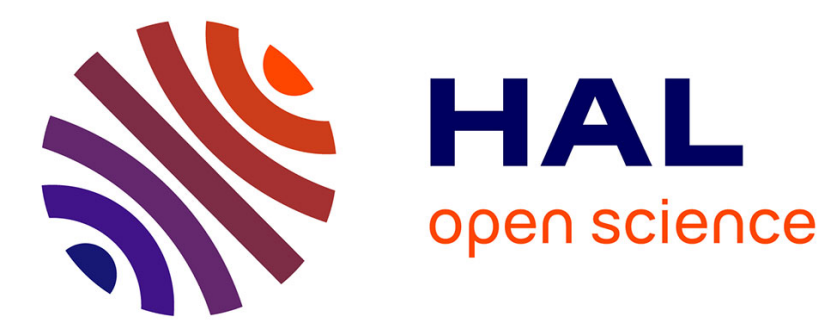

\title{
Skill Modelling for Digital Factories
}

\author{
Damiano Arena, F. Ameri, Dimitris Kiritsis
}

\section{To cite this version:}

Damiano Arena, F. Ameri, Dimitris Kiritsis. Skill Modelling for Digital Factories. IFIP International Conference on Advances in Production Management Systems (APMS), Aug 2018, Seoul, South Korea. pp.318-326, 10.1007/978-3-319-99707-0_40 . hal-02177838

\section{HAL Id: hal-02177838 \\ https://hal.inria.fr/hal-02177838}

Submitted on 9 Jul 2019

HAL is a multi-disciplinary open access archive for the deposit and dissemination of scientific research documents, whether they are published or not. The documents may come from teaching and research institutions in France or abroad, or from public or private research centers.
L'archive ouverte pluridisciplinaire HAL, est destinée au dépôt et à la diffusion de documents scientifiques de niveau recherche, publiés ou non, émanant des établissements d'enseignement et de recherche français ou étrangers, des laboratoires publics ou privés. 


\title{
Skill Modelling for Digital Factories
}

\author{
D. Arena ${ }^{1}$, F. Ameri ${ }^{2}$, D. Kiritsis ${ }^{1}$ \\ ${ }^{1}$ École Polytechnique Fédérale de Lausanne (EPFL), \\ SCI-STI-DK ME, Station 9, CH-1015 Lausanne, Switzerland \\ ${ }^{2}$ Texas State University, \\ Department of Engineering Technology, 601 University Dr., \\ San Marcos, TX 78666, USA \\ \{damiano.arena, dimitris.kiritsis\} depfl.ch \\ amerietxstate.edu
}

\begin{abstract}
In the past two decades, the use of ontologies has been proven to be an effective tool for enriching existing information systems in the digital data modelling domain and exploiting those assets for semantic interoperability. Despite the presence of many databases for industrial skills and professions, a formal representation, namely, an ontology, which meets the requirements of an existing tool for skill and capability analysis called CaMDiF is missing. In this research, the MSDL ontology, used by the tool in its initial version, is extended by importing modules of upper level and mid level ontologies (BFO and Agent Ontology) and by developing a new ontology for industry skills and professions based on an existing non-ontological resource $\left(\mathrm{O}^{*} \mathrm{NET}\right)$. As a result, an overview of the enriched data structure is provided along with some discussions on a use case related to skill analysis enabled by the new CaMDiF's skill modelling features
\end{abstract}

Keywords: Capability $\bullet$ Skill Modelling $\bullet$ Ontology $\bullet$ Digital Factory

\section{Introduction}

The increasing adoption of ontologies in industry is motivated by the need for enhancing the intelligence and interoperability of various business solutions in the context of Industry 4.0 paradigm. In particular, there is an emerging need for formal representation of industrial skills and professions in order to properly take into account the human resources while performing capability analysis for digital factories. In presence of formal and machine-interpretable models of human skills and profession, it is possible to conduct in-depth search and analysis on the available skills in a single factory or a group of factories and identify the areas of strength and weakness in terms of competencies.

adfa, p. 1, 2011.

(C) Springer-Verlag Berlin Heidelberg 2011 
Human skill is a complex, evolving, and multifaceted entity. To properly represent the semantics of human skills, a high level of expressivity and extensibility is called for. Ontologies, due to their logic-based underpinning, provide the required level of expressivity, formality, and flexibility for skill modelling.

Ontology Engineering refers to "The set of activities that concern the ontology development process and the ontology lifecycle, the methods and methodologies for ontology building, and the tool suites and languages that support them" [1].

The employment of ontology engineering technologies in the area of industrial data and information modelling has opened the path for exploiting ontologies towards providing formal definitions of the elements and their types, properties and interrelationships that exist for the domain of discourse [2]. An extensible ontology dealing with worker competencies was proposed in [3]. In this work, the current state of the so-called General Competency Schema (GCS) is presented. This is an OWL ontology that aims to provide a representation for requirement relationships between competencies and their constituents that to enable inference of competencies. It also provides several metrics for competency measurement and for specifying levels of required competencies. A rather simple ontological model for representing, inferring, and validating competencies over time is presented in [4] as an extension of the Process Specification Language (PSL). The authors provide a detailed range of axioms and inference rules expressed in First Order Logic (FOL), moreover, addressing a non-trivial subject such as the reliability of the information sources for worker skills. In the first paper, authors state that the model is flexible and can be easily adapted to the organization need, however, the organization is not mentioned in the model, and thus, it is unclear what role it may play. Much the same applies to the second work where, again, the worker's context is not taken into account.

The current research aims to extend an existing tool for capability analysis of digital factories by introducing a worker-centred ontology-based module for skill and profession modelling. The document is structured as follows. Section 2 discusses the existing works and their relations with the current research. Section 3 introduces the proposed skill ontology and its modules. In Section 4, the extension of CaMDiF based on the skill ontology and two use cases are discussed briefly.

\section{Relation to current research}

\subsection{CaMDiF}

CaMDiF (Capability Modeling for Digital Factory) is a software framework that is used for ontological representation and analysis of capabilities of digital factories. The Digital Factory, as the digital twin of a physical factory, replicates the facility in terms of installed machinery, material handling equipment, human resources, and other production support systems, including both software and hardware. 
The CaMDiF framework has a three-level architecture. The main components of the data and knowledge layer are the capability ontology and the manufacturing capability thesaurus, external domain ontologies, and the libraries of manufacturing resources including CNC machine and 3D printer, factory, and supply chain libraries. The second layer is the semantic layer which is basically the Apache Jena semantic application suite which provides a set of Java libraries and Application Programming Interfaces (APIs). Jena allows programmers to create, edit, and manage semantic web ontologies using RDF graphs. Also, Jena provides the necessary interfaces for query and reasoning that are usually needed in semantic applications. The last layer is the application layer that has three main functions, namely, build, analyse, and match. The first implementation of CaMDiF does not address human skills as part of the company's capability model. This paper introduces an extension of CaMDiF in which enables manufacturing companies to add the capabilities offered by human resources to their company profiles.

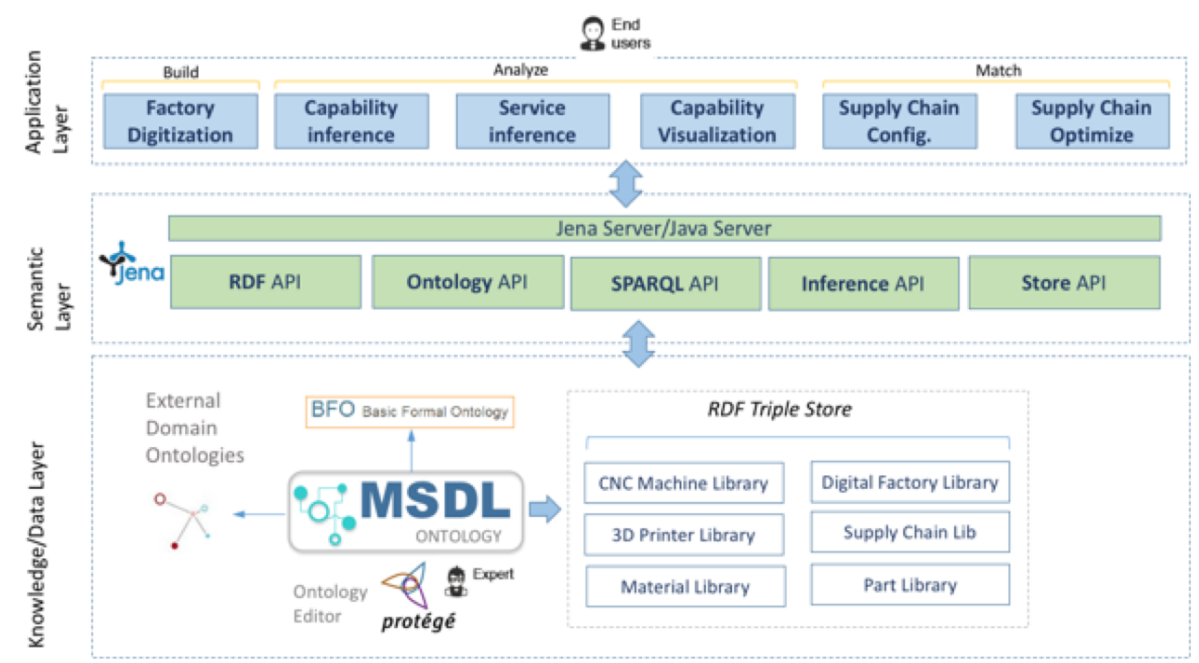

Fig. 1. The architecture of the CaMDiF Framework

\subsection{Profession and Skill Databases}

A non-exhaustive list of the existing ontological and non-ontological resources (structured models, guidelines, etc.) is presented in Table 1 and described below.

ESCO is a wide classification by the European Commission for Skills, Competencies, Qualifications and Occupations. Available in TTL and XLS formats, it is of particular interest regarding transversal skills/competencies.

The International Standard Classification of Occupations is a universal mono-hierarchical classification scheme of occupation groups, compiled by the International Labour Organization (ILO). There have been published four versions of the classification 
since 1957, abbreviated to ISCO-58, ISCO-68, ISCO-88 and ISCO-08, respectively. Among others, the ISCO classification is used by the European Union and by its member nations as a basic scheme for the construction of national occupation classifications.

ASOC stands for the Arab Standard Occupational Classification and is known as Tasneef in Arabic. It is the occupational classification system that is being built in the Kingdom of Saudi Arabia in 2015. ASOC covers all occupations and jobs in the national economy, including occupations in the public and private sectors. The classification system is built based on ISCO-08 and is available in Arabic and English.

The Italian occupation classification Classificazione delle Professioni (CP) 2011 is based on ISCO-08 and available in two languages (IT, EN), latest version 2011.

The Occupational Information Network (O*NET) in the USA is supported by the US Department of Labor/Employment and Training Administration (USDOL/ETA). It is related to the UK occupation classification SOC. O*NET is a broad database of occupation descriptions and the respective skills, competencies, etc. needed for each of them. Each occupation is structured into Tasks, Tools used, Knowledge, Skills, Ability, Work Activities, Work Context, Job Zone.

The European Dictionary of Skills and Competencies (DISCO) II is an online thesaurus with currently more than 104,000 skill and competence terms and about 36,000 phrases in the fields of health, computing, social services, environmental protection and non-domain specific skills and competencies. Available in eleven European languages, DISCO is one of the largest collections of its kind in the education and labour market.

The Répertoire Opérationnel des Métiers et des Emplois (ROME), version 3, the French Occupation Classification has its own structure, thus it is not based on ISCO08. The structure contains three levels: occupation categories, fields of work and the occupation term, to which all the occupations are assigned.

Table 1. Comparison of the existing skill databases

\begin{tabular}{|c|c|c|c|c|c|c|}
\hline \multirow{2}{*}{ Resource } & \multicolumn{4}{|c|}{ Format } & \multirow{2}{*}{ Documented } & \multirow{2}{*}{ Language(s) } \\
\hline & TTL & JSON & XLS & TXT & & \\
\hline ESCO & $\mathrm{x}$ & & $\mathrm{x}$ & & Yes & English \\
\hline ISCO-08 & & & & $\mathrm{x}$ & Yes & English \\
\hline ASOC & & & $\mathrm{x}$ & & Yes & Arabic, English \\
\hline CP 2011 & & & & $\mathrm{x}$ & Yes & Italian, English \\
\hline O*NET & & & $\mathrm{x}$ & $\mathrm{x}$ & Yes & Multiple \\
\hline DISCO II & & & & $\mathrm{x}$ & Yes & English \\
\hline ROMEV3 & & $\mathrm{x}$ & $\mathrm{x}$ & & Yes & French \\
\hline
\end{tabular}




\section{$3 \quad$ Ontology Modelling}

The ontology used in this work has different modules, namely, BFO, Agent Ontology, MSDL, and ISPO, which are described in following sub-sections.

\subsection{Description of BFO}

Basic Formal Ontology (BFO) is used as the foundational, or upper, ontology in this work [5]. As a domain-neutral upper-level ontology, BFO adopts a view of reality and represents different types of entities that exist in the world and relations between them. BFO is deliberately designed to be very small and its most recent version, BFO 2.0, has 35 classes. There are two types of entities in BFO, namely, Continuants and Occurrents. Continuants are the entities that continue to persist through time while maintaining their identity whereas, Occurrents are the events or happenings in which Continuants participate. Apart from its realistic approach, BFO has multiple other unique features that make it an appropriate upper ontology for many domains. Firstly, BFO has a very large user base and it is widely used in a variety of ontologies. Secondly, BFO is very small and therefore, easy to use and easy to learn. Additionally, BFO is very welldocumented and there are multiple tutorials, guidelines, and web forums for using BFO in ontological projects.

\subsection{Description of MSDL}

The capabilities of a Digital Factory are formally described using the MSDL ontology. Manufacturing Service Description Language (MSDL) is an ontology for representation of capabilities of manufacturing services. MSDL decomposes the manufacturing capability into four levels of abstraction, namely, company-level, shop-level, machinelevel, and device-level [6]. MSDL was initially developed to enable automated supplier discovery in distributed environments with focus on mechanical machining services but the classes were intentionally designed to be generic enough to address a wide range of manufacturing processes and services.

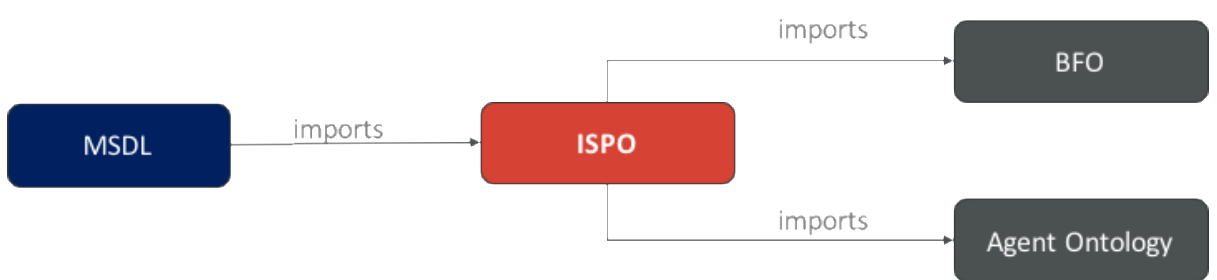

Fig. 2. Ontology network 


\subsection{Description of ISPO}

Despite the existence of an ontological resource called ESCO, which provides a Turtle ontology-based classification for skills, distinguishing between skill/competence and knowledge elements by indicating the skill type, in this work, we present an ontological model generated from a non-ontological resource named O*NET, the structure of which better suits the needs of the new CaMDiF architecture presented in Section 3.1.

Starting from a list of unstructured information organized within an XSL document, an ad hoc MatLab@ script has been designed and used to generate the class hierarchy based on O*NET's structure. Fig. 3 shows the first two class levels of the Industry Skills and Professions Ontology (ISPO).

At its present state of development, the ISPO Ontology only contains is a relations and serves as a bridge between the MSDL and both BFO and Agent Ontologies Fig. 2. As a result, the ontology network (Fig. 4) exploits parts of MSDL, BFO, ISPO, and Agent Ontology for semantic data annotation, interpretation, and querying.

\subsection{Description of Agent Ontology}

ISPO and MSDL both import the Agent Ontology in order to represent various agents and the roles they have in different contexts. The Agent Ontology is one of the eleven mid-level ontologies that together comprise the Common Core Ontologies (CCO). In the Agent Ontology, the class Agent is defined as "An Independent Continuant that is capable of performing Intentional Acts". The class Agent can represent both individual agents (Person) and coordinated groups of individuals (Organization). Another core class of the Agent Ontology that is reused in the present work is the Occupation Role class that is defined as "A Role that inheres in an Agent in virtue of the responsibilities that Agent is expected to fulfil within the context of some Act of Employment".

For example, a Factory Operator, as an individual agent, is a person that assumes the role of Operator Role, as a sub-class of Occupation Role. Agents can have both qualities and capabilities. The Agent Capability class is defined as a "Realizable Entity that inheres in an Agent to the extent of that Agent's capacity to realize it in Intentional Acts of a certain type". In the Agent Ontology, Skill, as a defined class in Agent Ontology, is an agent capability that inheres in a Person. The Agent Ontology does not provide any subclass of the Skill and Occupation Role classes. Therefore, ISPO extends the Skill and Occupation Role classes in order to cover a wide range of skills and professions in manufacturing organizations. 


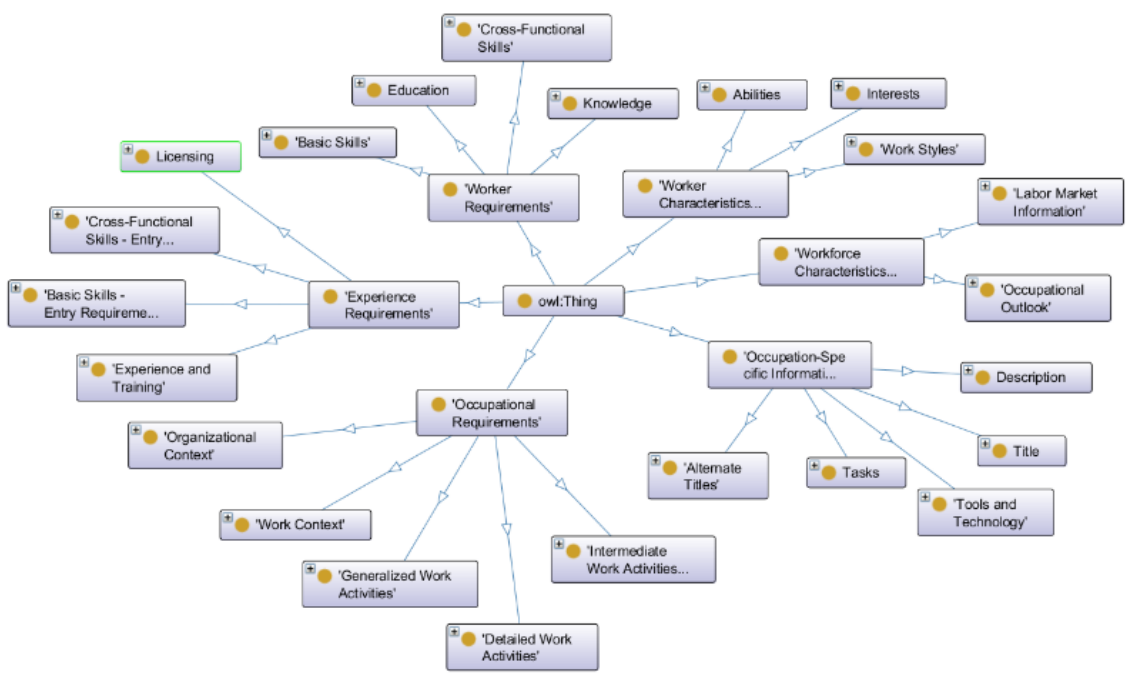

Fig. 3. First two class levels of ISPO

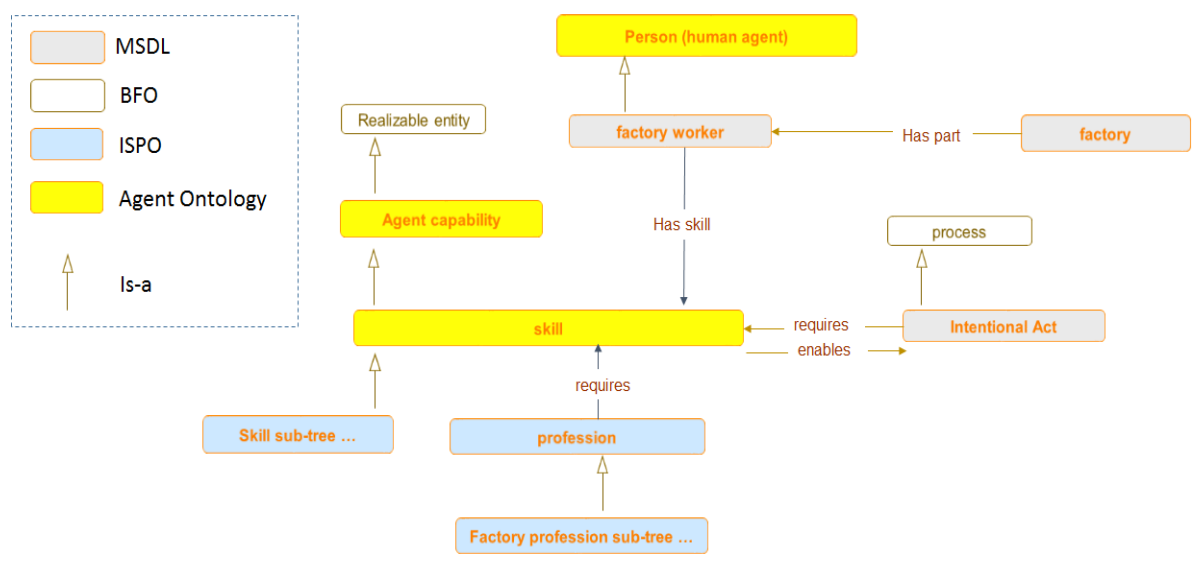

Fig. 4. Top-level class diagram of the ontology

\section{CaMDiF Extension with skill modelling feature}

The use of the abovementioned ontological models allows the extension of CaMDiF tool with skill modelling features. It enables semantics-driven capability and skill gap analysis for digital factories. Fig. 5 shows the user interface implemented for addition of skills to the capability model of the digital factory in CaMDiF. Since Skill is a subclass of the Disposition class in BFO, its level should be measurable along some scale. In this work, an ordinal scale (low-medium-high) is chosen for specify the level of each available type of skill. 


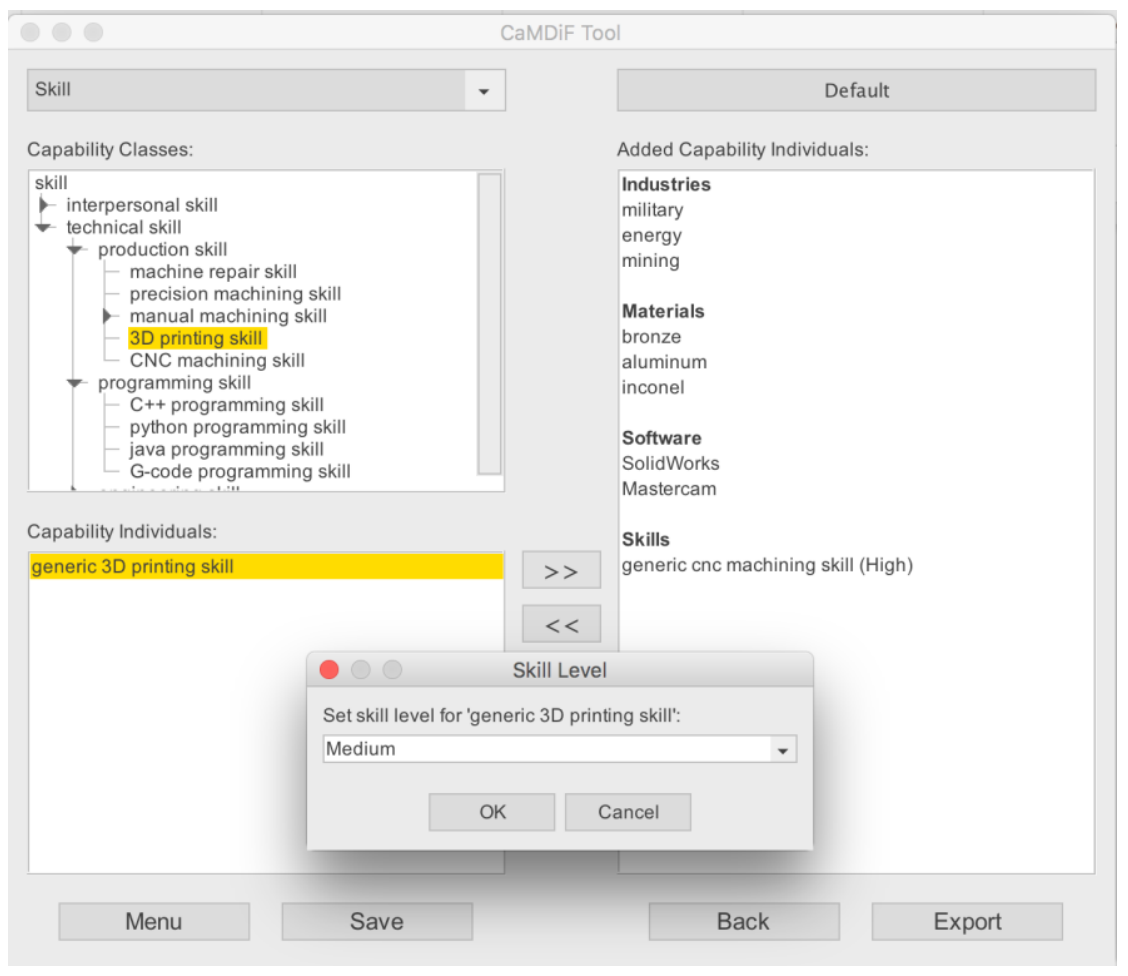

Fig. 5 The screenshot of the skill selection interface in CaMDiF

One utility of including skills ontology in CaMDiF is performing skill gap analysis. Skill gap analysis can be carried out through comparing the required skills for a particular manufacturing sector with the provided skills by the factories in CaMDiF's factory repository. The required skills can be obtained from the historical work orders available in the CaMDiF's work order repository. For example, CaMDiF's work orders can be queried to find the top ten skills required by all work orders in the aerospace sector. If the scope of the gap analysis is a single factory, then the gaps can be identified through subtracting the provided skills, by the factory, from the requested skills in the aerospace sector. The scope of gap analysis can be extended to cover all factories that provide precision machining services, for example. The identified skill gap informs the precision manufacturing sector about the new skills that need to be acquired in order to better serve the needs of customers from the aerospace industry.

In the current implementation of $\mathrm{CaMDiF}$, the required skills for a given work order are asserted explicitly. However, in the future versions, it is possible to implement a mechanism for inferring the required skills for a given work order based on the part properties such as material, tolerances, and surface finishes as well as the required manufacturing processes for fulfilling the order. 


\section{Conclusions}

In this paper, a new ontology for representation of manufacturing skills and professions was introduced and it was demonstrated how the ontology can be used for skill gap analysis in CaMDiF framework. The main contribution of this paper is to propose a modular, BFO conformant ontology for skill representation. The skill ontology can be used for formal description of the capabilities of digital factories. Formal skill representation is a major enabler for competency-based supply chain formation solution. The layered and modular structure of the ontology allows for seamless extension of the ontology in different sub-domains of manufacturing industry. One of the research issues that needs to be addressed in the future is automated extraction of required skills for manufacturing work orders.

\section{Acknowledgement}

This work was partially supported by the EU funded DILECO project (ID 785367).

\section{$7 \quad$ References}

1. Kiritsis, Dimitris. "Closed-loop PLM for intelligent products in the era of the Internet of things." Computer-Aided Design 43.5 (2011): 479-501.

2. Arena, Damiano, et al. "Human resource optimisation through semantically enriched data." International Journal of Production Research (2017): 1-23.

3. García-Barriocanal, Elena, Miguel-Angel Sicilia, and Salvador Sánchez-Alonso. "Computing with competencies: Modelling organizational capacities." Expert Systems with Applications39.16 (2012): 12310-12318.

4. Fazel-Zarandi, Maryam, and Mark S. Fox. "An Ontology for Skill and Competency Management." FOIS. 2012.

5. Arp, Robert, Barry Smith, and Andrew D. Spear. Building ontologies with basic formal ontology. Mit Press, 2015.

6. Ameri, Farhad, and Debasish Dutta. "An upper ontology for manufacturing service description." ASME 2006 international design engineering technical conferences and computers and information in engineering conference. American Society of Mechanical Engineers, 2006. 